\title{
APLICAÇÃO DE PROCESSO TERMOQUÍMICO COMO APROVEITAMENTO DE RESÍDUO AGROINDUSTRIAL DE BABAÇU
}

\author{
G. E. G. VIEIRA ${ }^{1}$, A. PICKLER ${ }^{2}$, L. F. P. GALLO ${ }^{2}$, L. F. TEIXEIRA ${ }^{1}$, A. G. N. COLEN ${ }^{1}$, \\ C. E. A. CAMPOS ${ }^{1}$, G. L. de AGUIAR Jr. ${ }^{1}$ e R. C. da SILVA $^{1}$ \\ ${ }^{1}$ Universidade Federal do Tocantins (UFT), Laboratório de ensaio e desenvolvimento em \\ biomassa e biocombustíveis (LEDBIO) \\ ${ }^{2}$ CENPES - PETROBRAS \\ E-mail para contato: glauciaeliza@pq.cnpq.br
}

\begin{abstract}
RESUMO - No contexto atual de crescentes preocupações ambientais, a valorização de resíduos agroindustriais do ponto de vista energético é de grande interesse. Dentre as formas de aproveitamento dos resíduos, os processos termoquímicos possibilitam a obtenção de produtos com potencial e propriedades energéticas, dentre elas, o processo de pirólise permite a obtenção de quatro frações: bio-óleo, aquosa, sólida e gasosa. O babaçu possui uma das cadeias do extrativismo vegetal mais representativas do Brasil. No presente trabalho, foi realizada a caracterização físico-química e aplicação do processo de pirólise ao resíduo torta de babaçu, resultante da extração de óleo do babaçu, utilizando as temperaturas de processo de $550^{\circ} \mathrm{C}$ e $450^{\circ} \mathrm{C}$, taxa de aquecimento de $10^{\circ} \mathrm{C} / \mathrm{min}$ e tempo de retenção de 120 minutos, para cálculo de rendimento das frações. Os resultados obtidos na caracterização foram de $3,42 \%$ de umidade, $92,32 \%$ de voláteis, $3,93 \%$ de cinzas e $0,33 \%$ de carbono fixo. O processo de pirólise apresentou rendimento de 18,41\% e 18,50\% de bio-óleo, 14,39\% e $11,79 \%$ de fração aquosa, $29,89 \%$ e $32,50 \%$ de fração sólida, $37,31 \%$ e $37,21 \%$ de fração gasosa, respectivamente nas temperaturas de $550^{\circ} \mathrm{C}$ e $450^{\circ} \mathrm{C}$.
\end{abstract}

\section{INTRODUÇÃO}

O Brasil tem um grande potencial para o uso de matérias-primas renováveis. É um dos maiores produtores de commodities agrícolas e culturas extrativistas, produzindo grandes quantidades de agroresíduos. Estes resíduos podem ser efetivamente transformados em energia e outros produtos, em processos integrados envolvendo a conversão da biomassa em combustíveis, energia e produtos químicos (Foster-Carneiro et al., 2013).

A identificação, avaliação e controle dos impactos ambientais das atividades agroindustriais devem ser estimulados visando à redução dos impactos decorrentes da disposição inadequada dos resíduos gerados. A Resolução Conama no 316/2002, por sua vez, dispõe sobre os resíduos agrícolas e da silvicultura que possuem potencial para serem encaminhados para tratamento térmico, além da Lei Federal no 8.171/91 (Brasil, 1991) que prevê o aproveitamento dos resíduos da agricultura.

Os termos resíduos agrícolas e resíduos agroindustriais possuem um amplo significado, referindo-se a qualquer resíduo de natureza lignocelulosica produzido pela prática agrícola ou 
agroindustrial em suas operações diárias, tais como folhas, raízes, talos, cascas, bagaço, resíduos de palha, sementes, resíduos de madeira e resíduos de origem animal. Todos estes resíduos representam uma fonte de bilhões de toneladas por ano, em grande parte disponível e renovável, constituindo uma categoria importante com alto potencial de aproveitamento, aliado ainda ao fato de não competirem com a disponibilidade de alimentos (Taherzadehe; Karimi, 2007).

A abrangência da palmeira babaçu é de aproximadamente 13 a 18 milhões de hectares em 279 municípios, situados em 11 Estados, com inúmeras potencialidades e atividades econômicas desenvolvidas a partir de sua incidência (Carrazza et al., 2012). Em 2011 foram extraídas 102.499 toneladas de amêndoas de babaçu no Brasil, que girou um capital de cerca 142 milhões (IBGE, 2012). A amêndoa do babaçu é o segundo produto florestal não madeireiro mais vendido no Brasil, o valor da venda dessa produção chega a ultrapassar o do açaí (produto não madeireiro mais vendido no país), alcançando quase R\$ 150 milhões. Sua cadeia produtiva é uma das mais representativas do extrativismo vegetal no Brasil (Carrazza et al., 2012).

Em termos de produção, a cadeia produtiva do babaçu é relativamente simples. $\mathrm{Na}$ prensagem da amêndoa do babaçu para obtenção de óleo, principal produto comercial do babaçu, tem-se o rendimento médio de $4,6 \%$ de óleo de babaçu e $2,4 \%$ de torta (Santos, 2008).

A produção de energia a partir de biomassa pode ser dividida em duas categorias principais: processos termoquímicos e vias de conversão biológica. Existem diversas rotas termoquímicas para produção de energia, dentre elas a pirólise (Balat et al., 2009).

No processo pirolítico são gerados produtos com potencial fonte de combustíveis ou usos relacionados à indústria (Karayildirim et al., 2006). Essa possibilidade de obter produtos com valor agregado torna esse processo uma opção diante das iniciativas atuais de busca de novas fontes renováveis para a produção de energia limpa (Gómez, 2002). Durante a pirólise são obtidos produtos gasosos, líquidos e sólidos, que são gerados em diferentes proporções a partir do rompimento térmico das ligações, obtenção de produtos com densidade energética mais alta e melhores propriedades do que àquelas da biomassa inicial (Vieira, 2004).

Neste estudo foram realizadas análises de teor de umidade, voláteis, cinzas e carbono fixo de torta de babaçu, a fim de conhecer as características deste agroresíduos, e foi aplicado o processo de pirólise de diferentes temperaturas de processo obtendo-se quatro frações: bioóleo, aquosa, sólida e gasosa.

\section{MATERIAIS E MÉTODOS}

\subsection{Aquisição e preparo analítico da amostra}

A torta de babaçu utilizada no presente trabalho foi doada pela empresa TOBASA Bioindustrial de Babaçu S/A, localizada no município de Tocantinópolis, no Estado do Tocantins, gerada a partir do processamento agroindustrial do coco babaçu. Foi classificada 
de acordo com a norma NBR 10.004 em resíduos da classe II A - não perigoso e não inerte, e o procedimento de amostragem foi realizado segundo a norma NBR 10.007.

A amostra de torta de babaçu foi triturada em moinho e homogeneizada através de peneiras do sistema da Tyler (partículas de aproximadamente 1,7 mm), com a finalidade de facilidade os procedimentos analíticos. A Figura 1 mostra a amêndoa do coco babaçu, a torta proveniente da prensagem da amêndoa e a amostra de torta de babaçu triturada e homogeneizada.
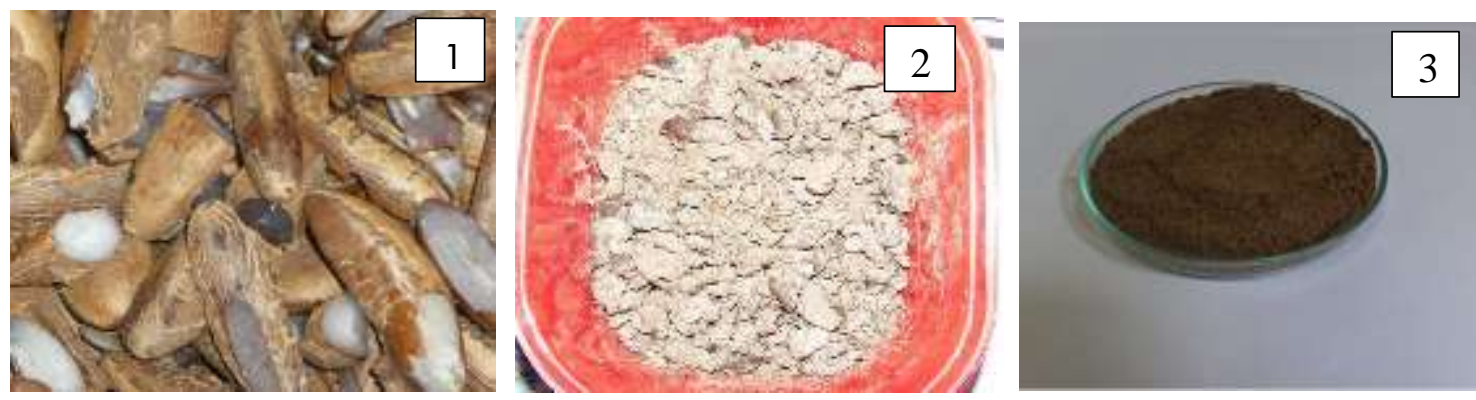

Figura 1 - Amêndoa do coco babaçu (1); Torta de babaçu (2); Amostra de torta de babaçu triturada (3)

\subsection{Análises imediatas}

Caracterizou-se a amostra de torta de babaçu através da determinação do teor de umidade, voláteis, cinzas e carbono fixo, segundo metodologia descrita pela Tabela 1.

Tabela 1 - Metodologias para análises imediatas

\begin{tabular}{|c|c|}
\hline Analise & Métodos \\
\hline Umidade & ASTM D3173-85 \\
\hline Voláteis & ASTM D2415-66 \\
\hline Cinzas & ASTM D2415-66 \\
\hline Carbono fixo & Diferença \\
\hline
\end{tabular}

\subsection{Processo termoquímico}

O processo de pirólise termoquímica foi realizado em escala laboratorial, em reator de leito fixo da marca EDG, constituído pelos seguintes sistemas: tubo de cerâmica, tubo reator de quartzo, sistema de alimentação de gás inerte (nitrogênio), sistema de condensação, sistema de separação de líquidos ou bio-óleo e lavadores de gases. Os ensaios foram realizados em duplicata, utilizando 40 gramas de amostra. As condições do processo aplicadas à biomassa em estudo estão descritas na Tabela 2.

Tabela 2 - Condições de processo utilizadas na pirólise

\begin{tabular}{|c|c|c|c|}
\hline \multirow{2}{*}{ Amostra } & $\begin{array}{c}\text { Temperatura } \\
\left({ }^{\circ} \mathbf{C}\right)\end{array}$ & $\begin{array}{c}\text { Tempo de } \\
\text { retenção }(\mathbf{m i n})\end{array}$ & $\begin{array}{c}\text { Taxa de Aquecimento } \\
\left({ }^{\circ} \mathbf{C} / \mathbf{m i n}\right)\end{array}$ \\
\hline \multirow{2}{*}{ Torta de babaçu } & 450 & 120 & 10 \\
\cline { 2 - 4 } & 550 & & 10 \\
\hline
\end{tabular}




\section{RESULTADOS E DISCUSSÃO}

O coco babaçu é dividido em quatro partes: epicarpo, mesocarpo, endocarpo e amêndoa. Sua capacidade de fornecer uma ampla variedade de produtos úteis. A Tabela 3 mostra a composição típica dos cocos babaçu da cidade de Tocantinópolis (TO).

Tabela 3 - Composição típica do coco babaçu - Tocantinópolis - TO

\begin{tabular}{|c|c|}
\hline Peso médio do coco & $\mathbf{2 3 0} \mathbf{g}$ \\
\hline Epicarpo & $12 \mathrm{~g}$ \\
\hline Mesocarpo & $23 \mathrm{~g}$ \\
\hline Endocarpo & $58 \mathrm{~g}$ \\
\hline Amêndoas & $7 \mathrm{~g}$ \\
\hline
\end{tabular}

Fonte: Pavlak et al., (2007)

A caracterização físico-química por meio da determinação dos teores de umidade, sólidos voláteis, cinzas, carbono fixo é de grande importância em processos térmicos. A Tabela 4 apresenta os valores médios para a amostra de torta de babaçu.

Tabela 4 - Teores médios em percentagem das análises imediatas de torta de babaçu

\begin{tabular}{|c|c|}
\hline Analise & $\mathbf{\%}(\mathbf{m} / \mathbf{m})$ \\
\hline Umidade & 3,42 \\
\hline Voláteis & 92,32 \\
\hline Cinzas & 3,93 \\
\hline Carbono fixo & 0,33 \\
\hline
\end{tabular}

*Teor de carbono fixo obtido por diferença

Segundo Carrazza et al. (2012) e Silva (2009), o teor de umidade da torta de babaçu encontra-se aproximadamente em $11,5 \%$, sendo o teor máximo de umidade na torta resultante do processo de prensagem e extração por solvente químico de $12 \%$. O baixo valor de umidade encontrado na amostra de torta de babaçu em estudo $(3,42 \%)$ pode estar relacionado ao processo de extração de óleo utilizado pela indústria a qual foi fornecido.

Dentre os compostos orgânicos que compõe a fração dos sólidos voláteis estão os materiais lignocelulósicos, como a celulose, hemicelulose, lignina, ácidos húmicos e ácidos carboxílicos (Pedroza, 2011). Silva (2009), analisou a torta de babaçu e apresentou 87,56\% de matéria orgânica, desse teor apresentou cerca de 31,98 \% de hemicelulose, $21,98 \%$ de celulose e $12,24 \%$ de lignina. O teor de voláteis do presente estudo foram superiores ao de agroresíduos como casca de coco $(67,9 \%)$, palha de arroz $(65,47)$ e palha de cana $(73,78 \%)$ (Teixeira, 2002; Vale et al., 2007).

Para aplicação da matéria-prima em processos de pirólise o teor de cinzas é de grande interesse, pois pode estar relacionado ao rendimento das frações no processo. O elevado teor de cinzas favorece reações com desprendimento de compostos voláteis em menores temperaturas, ocasionando formação de fração gasosa (Vieira, 2004; Fonts et al., 2009). O valor do teor de cinzas foi aproximado ao encontrado por Castro $(2012)$, de $4,49 \%(\mathrm{~m} / \mathrm{m}) \mathrm{em}$ torta de babaçu. 
As características finais dos produtos obtidos em processos de pirólise possuem dependência em grande parte das propriedades físico-químicas da matéria-prima utilizada, por exemplo, grande parte da fração volátil é formada a partir da celulose e da hemicelulose. Por outro lado a lignina contribui para a formação da fração sólida resultante do processo de pirolise (Mohan et al., 2006).

O processo de pirólise designa a decomposição térmica, consistindo em um processo termoquímico, ou seja, as transformações ocorrem em função da influência da temperatura e de reações químicas (Vieira, 2004). No processo de pirólise foram obtidas quatro frações: aquosa, bio-óleo, sólida e gasosa, a Figura 2 apresenta as frações bio-óleo, aquosa e sólida obtidos no processo de pirólise de torta de babaçu.

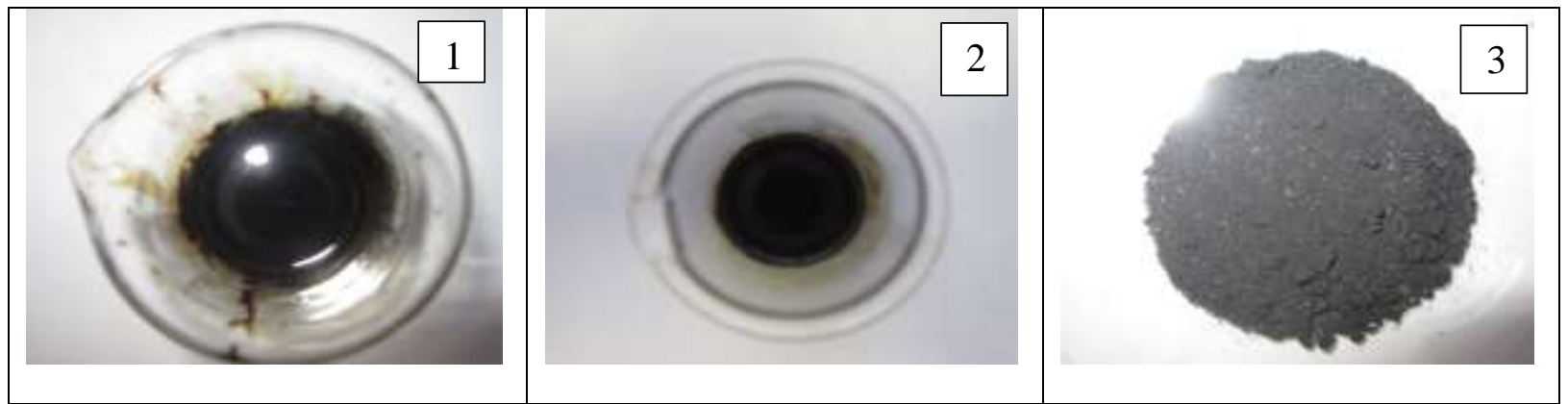

Figura 2 - Frações bio-óleo (1), aquosa (2) e sólida (3) obtidas a partir da pirólise de torta de babaçu

Os rendimentos médios de frações da pirólise da amostra de torta de babaçu foram comparados pela análise de variância a um nível de $95 \%$ de confiança. Os rendimentos dos produtos das pirólises realizadas nesse trabalho podem ser visualizados na Tabela 3. Diferença significativa foi encontrada somente na fração sólida, enquanto que para o bio-óleo, aquosa e gasosa a diferença entre os dois tratamentos não foi significativa.

Tabela 3 - Rendimentos das frações do processo termoquímico de torta de babaçu em percentagem $(\%)(\mathrm{m} / \mathrm{m})$

\begin{tabular}{|c|c|c|c|c|c|c|}
\hline \multirow{2}{*}{ Frações } & \multicolumn{3}{|c|}{$\mathbf{4 5 0}^{\mathbf{o}} \mathbf{C}$} & \multicolumn{3}{c|}{$\mathbf{5 5 0}^{\mathbf{}} \mathbf{C}$} \\
\cline { 2 - 7 } & $\mathbf{1}^{\mathbf{a}}$ repetição & $\mathbf{2}^{\mathbf{a}}$ repetição & Média & $\mathbf{1}^{\text {a }}$ repetição & $\mathbf{2}^{\mathbf{a}}$ repetição & Média \\
\hline Bio-óleo & 19,25 & 17,75 & $18,50 \mathrm{~ns}$ & 17,90 & 18,93 & $18,41_{\mathrm{ns}}$ \\
\hline Aquosa & 11,34 & 12,25 & $11,79 \mathrm{~ns}$ & 13,46 & 15,31 & $14,39_{\mathrm{ns}}$ \\
\hline Sólida & 32,54 & 32,46 & $32,50^{*}$ & 29,77 & 30,01 & $29,89^{*}$ \\
\hline Gasosa & 36,88 & 37,21 & $37,21_{\mathrm{ns}}$ & 38,87 & 35,75 & $37,31_{\mathrm{ns}}$ \\
\hline
\end{tabular}

ns: não significativo a um nível de $5 \%$ de probabilidade pela análise de variância; * significativo a $5 \%$ de probabilidade pela análise de variância

Os rendimentos em bio-óleo apresentaram médias iguais a 5\% de significância pelo teste de variância nos dois experimentos de pirólise, sendo os rendimentos obtidos de 18,5\% e $18,4 \%$, para as temperaturas de $450^{\circ} \mathrm{C}$ e $550^{\circ} \mathrm{C}$, respectivamente. Santos (2013) pirolisou amostra de torta de tucumã nas temperaturas $300^{\circ} \mathrm{C}, 400^{\circ} \mathrm{C}, 500^{\circ} \mathrm{C}$ e obteve os respectivos rendimentos de bio-óleo 5,5\%,7,9\%, 6,29\% e fração aquosa 14,7\%, 14,24\%, 10,77\%. Em 
pirólise de torta de mamona, Santos (2013) obteve o rendimento máximo de bio-óleo de $16 \%$ a $400^{\circ} \mathrm{C}$ e $14,59 \%$ a $500^{\circ} \mathrm{C}$, já $600^{\circ} \mathrm{C}$ obteve $6,53 \%$ de bio-óleo.

Os rendimentos da fração sólida nos dois tratamentos apresentaram diferença significativa a 95\% de confiança pela análise de variância. Comparando as médias dos dois tratamentos, pode se observar que com o aumento da temperatura, o rendimento da fração sólida diminuiu. $\mathrm{O}$ decréscimo na fração sólida a $550^{\circ} \mathrm{C}$ pode ser atribuído a um aumento da volatilização dos hidrocarbonetos sólidos no carvão.

\section{CONCLUSÃO}

Os estudos de caracterização físico-química da torta de babaçu através das análises imediatas mostraram que o mesmo apresentou alto teor de sólidos voláteis $(93,32 \%)$, baixo teor de umidade na amostra $(3,42 \%)$ e teor de cinzas $(3,93 \%)$, sendo uma baixa umidade um parâmetro desejável na biomassa para aplicação em processo de pirólise.

No processo de pirólise foram obtidas quatro frações, sendo o bio-óleo, aquosa, sólida e gasosa. As análises estatísticas de variância mostraram que não houveram diferença significativa entre médias das frações bio-óleo, aquosa e gasosa. Como as médias dos dois tratamentos são estatisticamente iguais entre si, pode-se justificar o uso da temperatura de $450^{\circ} \mathrm{C}$ em vez da de $550^{\circ} \mathrm{C}$, podendo com isso evitar o gasto desnecessário de energia, uma vez que estas frações sejam desejadas.

\section{REFERÊNCIAS}

ABNT - ASSOCIAÇÃO BRASILEIRA DE NORMAS TÉCNICAS. NBR 10.004 Resíduos sólidos - Classificação. Rio de Janeiro, ABNT, 1987a.

ABNT, ASSOCIAÇÃO BRASILEIRA DE NORMAS TÉCNICAS. NBR 10.007 Amostragem de Resíduos. Rio de Janeiro: ABNT, 1987b.

AMERICAN SOCIETY FOR TESTING AND MATERIALS. D 2415-66. Standard Test Method for ash Determination, 1986.

AMERICAN SOCIETY FOR TESTING AND MATERIALS. D 3173-85. Standard Test of Humity, 1985.

BALAT, M. et al. Main routes for the thermo-conversion of biomass into fuels and chemicals. Part 1: Pyrolysis systems. Energy Conversion And Management, v. 50, p.3147-3157, 2009.

BRASIL. Presidência da República. LEI Nº 8.171/1991. Dispõe sobre a Política Agrícola. 1991.

CARRAZZA, L. R.; ÁVILA, J. C. C. e; SILVA, M. L. da. Manual tecnológico de aproveitamento integral do fruto e da folha do babaçu (Attalea spp.). 2. ed. Brasília: Instituto Sociedade, População e Natureza, p.68, 2012. 
CASTRO, K.J. Torta de babaçu: consumo, digestibilidade, desempenho, energia metabolizável, energia líquida e produção de metano em ruminantes. Tese (Doutorado em zootecnia). Universidade Federal de Minas Gerais. Belo Horizonte, 2012.

FONTS, I. et al. Physicochemical properties of product liquid from pyrolysis of sewage sludge. Energy \& Fuels, [s. I.], v. 23, p.4121-4128, 2009.

FOSTER-CARNEIRO, T.; BERNI, M. D.; DORILEO, I. L.; ROSTAGNO, M. A. Biorefinery study of availability of agriculture residues and wastes for integrated biorefineries. Resources, Conservation And Recycling, v. 77, p.78-88, 2013.

GÓMEZ, E. O. Estudo da pirólise rápida de capim elefante em leito fluidizado borbulhante mediante caracterização dos finos de carvão. 2002. $167 \mathrm{f}$. Tese (Doutorado) - Curso de Engenharia Agrícola, Departamento de Faculdade de Engenharia Agrícola, Universidade Estadual de Campinas, Campinas, 2002.

INSTITUTO BRASILEIRO DE GEOGRAFIA E ESTATÍSTICA IBGE. Produção da Extração Vegetal e da Silvicultura: Busca por Estados. 2009. Disponível em: <http://www.ibge.gov.br/estadosat/>. Acesso em: 02 dez. 2013.

KARAYILDIRIM, T.; YANIK, J.; YUKSEL, B.. Characterization of products from pyrolysis of waste sludges. Energy Fuel, v. 85, p.1498-1508, 2006.

MOHAN, D.; PITTMAN JUNIOR, C. U.; STEELE, P. H.. Pyrolysis of Wood/Biomass for Bio-oil: A Critical Review. Energy \& Fuels, ., v. 20, p.848-889, 2006.

PAVLAK, M. C. de M. et al. Aproveitamento da farinha do mesocarpo do babaçu (Orbignya martiana) para obtenção de etanol. Evidência, Joaçaba, v. 7, n. 1, p.7-24, 2007.

PEDROZA, M. M. Bio-óleo e Biogás da degradação termoquímica de lodo de esgoto doméstico em cilindro rotativo. 2011. 192 f. Tese (Doutorado) - Curso de Engenharia Química, Universidade Federal do Rio Grande do Norte, Natal, 2011.

RESOLUÇÃO CONAMA No 316/2002. Conselho Nacional de Meio Ambiente. Dispõe sobre procedimentos e critérios para o funcionamento de sistemas de tratamento térmico de resíduos, 2002.

SANTOS, J. R. de J. Biodiesel de babaçu: avaliação térmica, oxidativa e misturas binárias / João Pessoa: UFPB, 2008.

SANTOS, N. A. do V. Pirólise rápida de coprodutos do processo produtivo do biodiesel: efeito das condições de pirólise e caracterização dos produtos. Lavras - UFLA, 2013.

SILVA, R. F. da. Avaliação nutricional da torta de babaçu e sua Utilização em dietas para frangos de corte label Rouge. 2009. 83 f. Tese (Doutorado) - Curso de Ciência Animal, Universidade Federal de Goiás, Goiânia, 2009. 
TAHERZADEH, M. J.; KARIMI, K.. Enzymatic-based hydrolysis processes for ethanol from lignocellulosic materials: a review. Bioresources, ., v. 2, n. 4, p.707-738, 2007.

TEIXEIRA, M. A. Biomassa babaçu no Brasil. In: ENCONTRO DE ENERGIA NO MEIO RURAL, 4.,, Campinas. Proceedings .Campinas, 2002. p. 29 .40, 2002.

VALE, A. T.; GENTIL, L. V.; GONÇALEZ, J. C.; COSTA, A. F. Caracterização energética e rendimento da carbonização de resíduos de grãos de café e de madeira. Cerne, Lavras, v. 13, n. 4, p. 416-420, 2007.

VIEIRA, G. E. G. Fontes alternativas de energia - Processo aperfeiçoado de conversão térmica. 2004. 181 f. Tese (Doutorado) - Curso de Química Orgânica, Departamento de Instituto de Química, Universidade Federal Fluminense, Rio de Janeiro, 2004. 